\title{
BUILDING AND SOLVING MATHEMATICAL PROGRAMMING MODELS IN ENGINEERING AND SCIENCE by Enrique Castillo, Antonio J. Conejo, Pablo Pedregal, Ricardo Garcia, and Natalia Alguacil
}

A BOOK REVIEW

\author{
BRADFORD D. ALLEN \\ Florida Institute of Technology \\ Department of Mathematical Sciences \\ Melbourne, FL 32901 USA \\ E-mail: ballen@fit.edu
}

(Received April, 2002; Revised September, 2002)

Castillo, Conejo, Pedregal, Garcia, and Alguacil's book introduces aspiring and practicing mathematicians, engineers, and applied scientists to important aspects of constructing mathematical programming models. The methods presented in this text lead to optimal solutions of a wide variety of physical, economic, and engineering problems. The authors also discuss conditions leading to well-defined problems.

Providing a deep theoretical analysis is not the approach used in this text. The authors claim such an approach risks leaving the reader overly cautious about the mathematical details, uninformed about the motivation behind mathematical procedures, and unclear about where procedures lead. Instead, the authors use carefully selected illustrative examples to introduce the reader to the field of mathematical programming. The authors believe their examples help convey how ingenious and profound the methods of mathematical programming actually are, and help make the book more attractive and readable.

Mathematical programming involves finding a set of variables that, subject to certain constraints, maximize or minimize an objective function. If the objective function and constraints are all linear, then methods of linear programming may be applied. For many problems, the simplex method is the method of choice. This method was first developed by Dantzig in 1947 and, over the years, has become increasingly popular. The simplex method iteratively improves the objective function by moving from one feasible extreme point to another along the convex boundary defined by the linear constraints. The authors also discuss interior (or boundary) methods that visit points in the interior of the feasible region. These methods are based on nonlinear programming techniques developed in the 1960's and popularized with Karmarker's method in 1984.

Several classic models are presented in the text and used to introduce the reader to linear programming. For example, a transportation problem is discussed where various warehouses with certain supplies send a product to various destinations with certain demands. The solution minimizes the total transportation cost. A production scheduling problem is also discussed where a manufacturer produces a type of item with a demand that fluctuates with time. The manufacturer must decide what production schedule will meet the demand and minimize the sum of all costs including, for example, overtime costs during high production times, idle cost during low production times, and storage costs. A diet problem is presented 
that involves determining what amounts of different nutrients are needed to minimize the costs of a diet while satisfying certain nutrient conditions. A network flow problem is also presented where a transportation network (e.g. a pipeline, railroad, highway, communication system, etc.) carries a homogeneous commodity from source nodes through intermediate nodes to sink nodes in a way that minimizes the total cost.

In many situations, some variables in a linear programming model are restricted to the integers, or restricted as $0-1$ binary variables. These restrictions on variables make the problems much more difficult to solve. However, mixed-integer linear programming methods are often very effective tools to optimize mixed-integer programming models. Approaches such as the branch-bound and Gomory-cuts methods, where a series of linear programming solutions increasingly restricts the feasible region, are discussed.

Several interesting integer programming problems are presented. For example, the classic 0-1 knapsack problem is discussed. In this problem, a subset of items is selected from a set of useful items. The items are selected so that the total utility of the subset is maximized but the carrying capacity of the knapsack is not exceeded. The problem of identifying the relevant symptoms of a given set of diseases is also discussed. The goal here is to identify a minimal set of symptoms so that each of the diseases can be identified according to the symptom levels. Another type of integer problem discussed in the text is a model of capacitated facility locations. This type of problem deals with deciding where to locate facilities on a finite set of sites while taking into account fixed and variable costs and the needs of clients. The problem is solved by optimizing certain economic criteria.

Linear models are appropriate for a wide range of problems, but in many situations, linear models are too restrictive. These situations require that the nonlinear aspects of the physical and mathematical realities be considered. Instead of maximizing or minimizing a linear objective function with linear constraints, nonlinear programming models have nonlinear objective functions and/or one or more nonlinear constraints.

The authors discuss necessary optimality conditions for nonlinear programming models. In this regard, the Karush-Kuhn-Tucker (KKT) conditions are the most important theoretical results. The KKT conditions provide the fundamental ideas behind many computational algorithms. In particular, the KKT conditions give rise to stopping criteria in searches for locally constrained optima where the gradient is not necessarily equal to zero.

Constrained optimization algorithms include penalty methods where the original constrained problem is transformed into a sequence of unconstrained problems through the use of penalty functions. The advantage of dealing with a sequence of unconstrained problems rather than a single constrained problem is that each of the unconstrained problems can be solved efficiently and reliably. The authors present an exterior-point penalty method where the sequence of solutions contains only infeasible points, and an interior-point penalty method where the sequence of solutions contains only feasible points.

Duality plays a crucial role in linear programming theory. The authors generalize duality principles to convex nonlinear programming problems. Moreover, the authors discuss computational methods to approximate a primal solution by optimizing the appropriate dual problem which, in these cases, is a Lagrangian problem. The advantage of working with the dual is that in the majority of instances, the problem reduces to finding the maximum of a concave function over a simple convex set with no local maximum other than the global maximum.

A practical illustration of duality in a separable problem is presented where different producers compete in a marketplace to produce and sell a given commodity. The producers, however, have different cost functions and production constraints. Moreover, demand in every time period to a fixed horizon must be satisfied, and no storage capacity is available. Both primal and dual approaches to solving this problem are presented. A centralized or primal approach has a system operator who, using knowledge of production costs and constraints for every producer, provides the optimal level of production for all producers. On the other hand, the dual approach to this problem uses the property that the objective function 
is separable; that is, solving the dual problem requires solving each producer's own individual profit-maximization problem.

The authors allocation an entire chapter to the General Algebraic Modeling System which provides a computer platform to define, analyze and solve optimization problems. The authors chose this package over several others for no other reason than "the authors happen to be familiar with it." The package is free and available at www.gams.com. As with the modeling techniques presented in the text, the authors introduce the computer package with plenty of relevant examples.

While many important areas of mathematical programming are not covered in the text, the topics that are covered allow the reader to solve a wide variety of interesting and frequently encountered problems. The power of the methods for solving practical problems quickly becomes evident with the unique presentation of modeling techniques and the effective use of real-life examples. The text is very well suited as a professional reference or as a text for advanced mathematics or engineering courses.

Building and Solving Mathematical Programming Models in Engineering and Science

by Enrique Castillo, Antonio J. Conejo, Pablo Pedregal, Ricardo Garcia, and Natalia Alguacil

Publisher John Wiley \& Sons

Publication Year 2002

ISBN 0-471-150436

Price: $\$ 94.95$ 


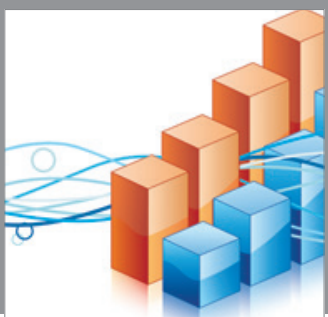

Advances in

Operations Research

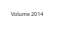

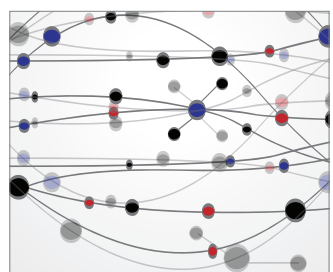

\section{The Scientific} World Journal
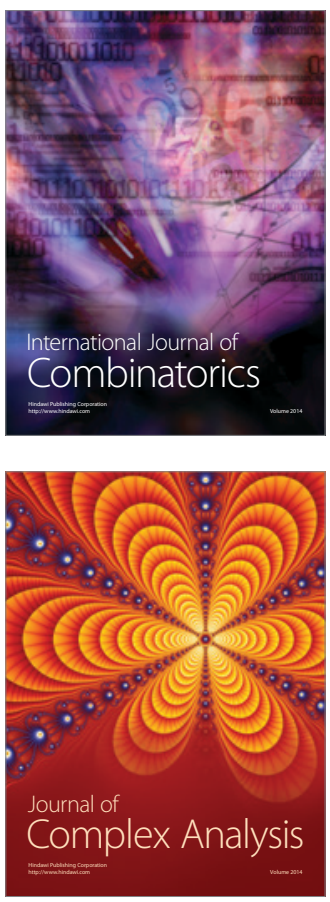

International Journal of

Mathematics and

Mathematical

Sciences
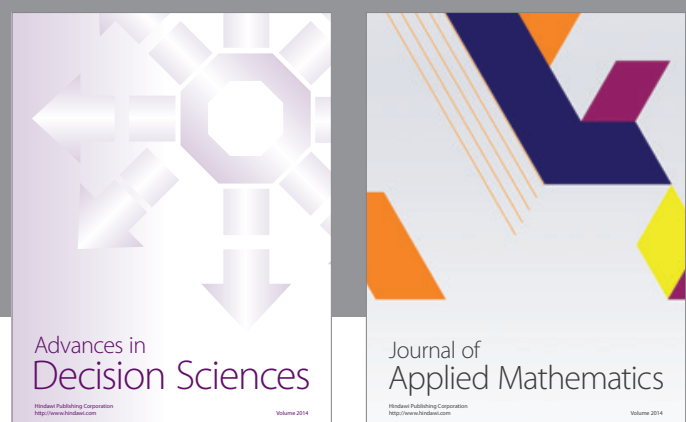

Journal of

Applied Mathematics
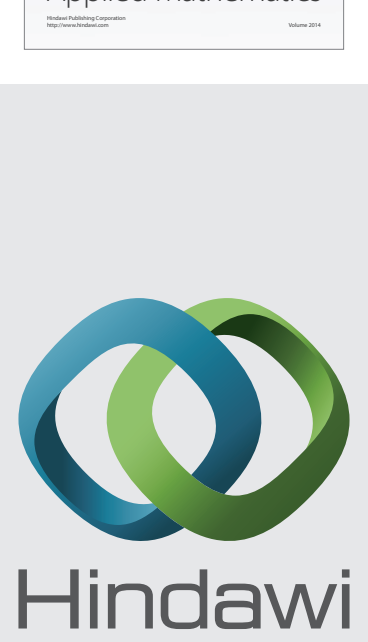

Submit your manuscripts at http://www.hindawi.com
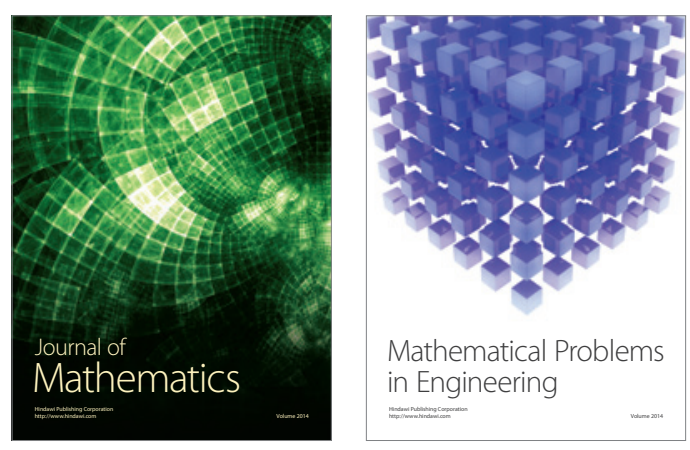

Mathematical Problems in Engineering
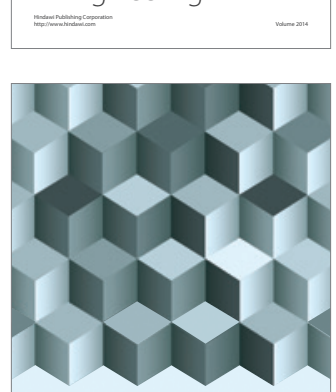

Journal of

Function Spaces
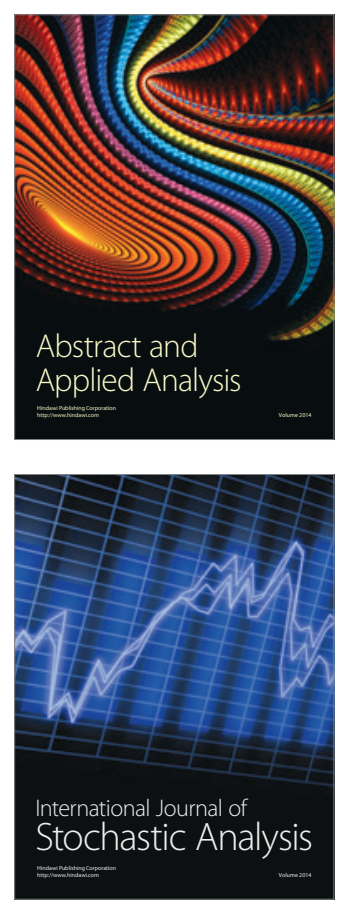

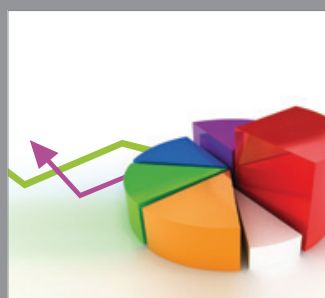

ournal of

Probability and Statistics

Promensencen
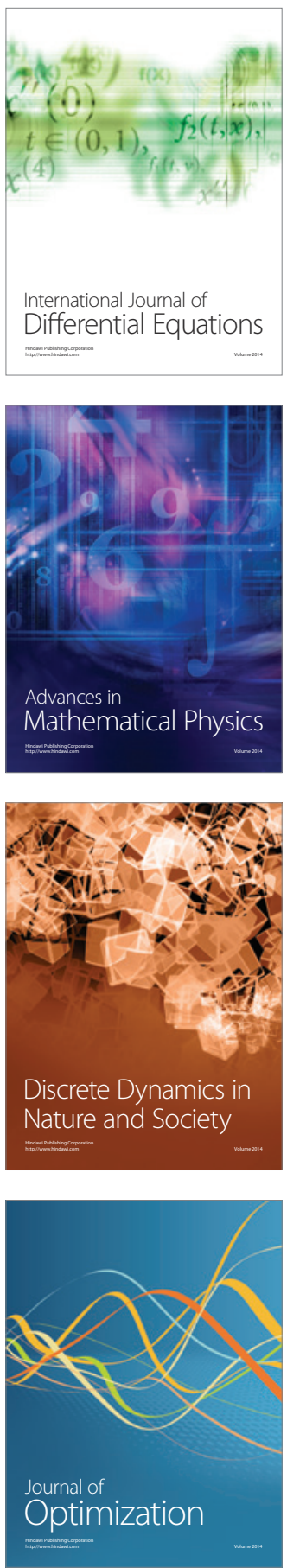